\title{
Analysis of Fire and Non-fire Signatures for Discriminating Fire Detection
}

\author{
JAMES A. MILKE and THOMAS J. McAVOY \\ University of Maryland \\ College Park, MD, USA
}

\begin{abstract}
The characteristics of an improved fire detector which promptly reacts to smoke while discriminating between airborne signatures from fire and non-fire sources can be identified by considering signature patterns of each group of sources. Discrimination is accomplished by comparing signature response patterns from fire and environmental sources collected in small- and large-scale tests. Airborne signatures are produced in the tests from a variety of conditions: flaming, pyrolyzing and heated samples, and nuisance sources, such as aerosols, household products and cooked food. The signatures are described in terms of light obscuration, temperature, $\mathrm{CO}, \mathrm{CO}_{2}, \mathrm{O}_{2}$ concentrations and signals from metal oxide sensors. An expert system was developed by a neural network and a multivariate statistical method to distinguish between fire and non-fire sources. In addition, the presence of a flaming or nonflaming fire can be identified despite the interjection of signatures from nuisance sources which could mask the fire signatures.
\end{abstract}

KEYWORDS: Smart fire detection, fire signatures, nuisance sources, non-fire signatures

\section{INTRODUCTION}

Prompt fire detection is the primary objective of automatic fire detection. The time to detection is a function of the sensitivity of the detector. However, a highly sensitive detector may provide a high frequency of unnecessary alarms because contemporary smoke detectors cannot discriminate between fire and non-fire sources of smoke and odors. Data from U.S. fire incidents during the 1980 's indicates that $95 \%$ of all alarms from smoke detectors were unnecessary [1]. One solution proposed by Thuillard for minimizing unnecessary alarms 
without sacrificing prompt activation involves using intelligence along with combinations of current sensor technology [2].

An interdisciplinary team from the Departments of Fire Protection Engineering and Chemical Engineering at the University of Maryland has conducted research to determine the characteristics of a sensitive, discriminating detector. The fire protection engineering team concentrated on selecting the fire and non-fire sources and characterizing the signatures from each source. The chemical engineering team applied analytical methods such as neural networks and multivariate statistical methods to investigate the signature and sensor response patterns and provide the discrimination capability between the flaming fire, non-flaming fire and non-fire sources. This effort has been conducted in three phases.

\section{SMALL-SCALE EXPERIMENTAL PROGRAM}

Initially, small-scale tests are conducted to characterize the signatures from fire and non-fire sources [3]. The experiments are designed to be conceptually similar to those by Okayama [4], with modifications incorporated to provide a greater range of measurements for describing the signature.

The small-scale experimental apparatus is a simplified tunnel with the airborne products of the sources introduced at a hood located at the inlet. Relatively elementary measurements are collected to provide a rudimentary view of the signatures. Measurements include temperature at the inlet and outlet of the apparatus. At the center of the apparatus, light obscuration, gas species concentrations $\left(\mathrm{CO}, \mathrm{CO}_{2}\right.$ and $\left.\mathrm{O}_{2}\right)$ and presence of any oxidizable gas are measured. The presence of oxidizable gases is measured by a Taguchi metal oxide sensor. Sources of the smoke or odor are placed under a hood at the inlet end of the apparatus. A variety of fuels and non-fire (nuisance) sources are selected to be representative of a residential environment. Airborne products are generated from a wide range of conditions: samples with flaming and pyrolyzing combustion, heated samples and aerosols.

An elementary expert system formulated from a manual review of the data is able to successfully classify 28 of 31 sources. The rules of the expert system are:

- $\mathrm{CO}_{2}$ concentration exceeds 1500 ppm only for flaming fires

- Peak CO concentration exceeds $28 \mathrm{ppm}$ and Taguchi detector response less than $6 \mathrm{~V}$ is acquired only for pyrolyzing solids.

- All other combinations are acquired from nuisance sources.

An ellipsoidal neural network is applied to the small-scale data, using data from two-thirds of the tests for training and the remainder for testing [5,6]. An improved classification rate is obtained, accurately classifying all sources except one smoldering source (which is improperly classified as a flaming source).

The level of success attained from the small-scale experimental program confirms the feasibility of the concept presented by Okayama. However, the success of the expert system 
and neural network only relates to the limited range of fuel sources investigated and the small-scale test apparatus.

\section{LARGE-SCALE EXPERIMENTAL PROGRAM}

In the second and third phases, large-scale experiments are conducted to determine whether the trends identified in the small-scale experimental effort are also applicable in large-scale environments. The large-scale experiments are conceptually similar to the small-scale experiments where signatures from a wide variety of fire and nuisance sources are monitored, with the sensor response patterns explored. In the second phase, either fire or non-fire sources are introduced alone. In contrast, in the third phase mixed sources including both fire and non-fire sources are provided simultaneously.

The large-scale experiments are conducted in a $3.6 \times 3.6 \mathrm{~m}$ room with a height of $2.4 \mathrm{~m}$ [79]. The room is unconditioned, with the temperature and humidity dictated by atmospheric conditions. Measurements include temperature, mass loss of the fire sources, $\mathrm{CO}, \mathrm{CO}_{2}$ and $\mathrm{O}_{2}$ concentrations, light obscuration and the voltage output from two metal oxide sensors (Taguchi models 822 and 880). In addition, two commercial smoke detectors (one photoelectric and one ionization) are located on the ceiling, at the center of the room. A diagram of the room, including the relative locations of the sensors, is provided as Figure 1. A detailed description of the instrumentation is provided elsewhere [7,9].

\section{FIGURE 1 Diagram of Test Room}

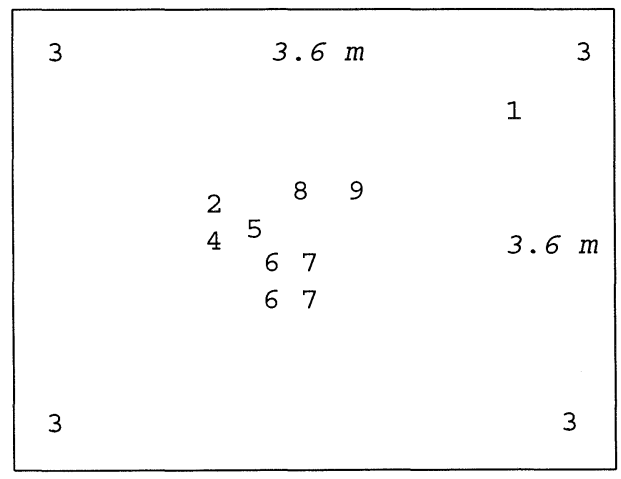

1. Load cell and fire source

3. Thermocouple tree

5. $4.75 \mathrm{~mm}$ copper sampling tube

7. Photoelectric smoke detector

9. Taguchi 880
2. Photocell

4. Helium-neon laser

6. Ionization smoke detector

8. Taguchi 822 
The Taguchi 822 and 880 metal oxide sensors are sensitive to the presence of oxidizable gases and environmental odors respectively. Mass loss measurements are used to estimate the yield fractions of the signatures from the fire sources. Yields of the non-fire sources are estimated based on the quantity of material introduced. Because the tests are conducted in an unconditioned space, data is collected for at least two minutes prior to introducing any source in order to document variations in ambient conditions.

\section{Single Source Experiments}

The variety of sources used in the second phase to generate conditions within the room are summarized in Table $1[7,8]$. The sources are intended to be representative of fire and nuisance sources in residential environments. The 87 tests involved introducing 34 flaming sources, 16 smoldering sources and 37 nuisance sources. In general, the flaming and smoldering fires were relatively small, i.e. the mass loss measured from flaming fires ranged from $0.008 \mathrm{~g} / \mathrm{s}$ for polystyrene to $1.99 \mathrm{~g} / \mathrm{s}$ for paper. Typically, the mass loss was on the order of $0.2 \mathrm{~g} / \mathrm{s}$.

\section{TABLE 1 Test Sources}

\begin{tabular}{|c|c|c|c|}
\hline Liquid & $\begin{array}{l}\text { Heated Fuels } \\
\text { Solid }\end{array}$ & Gas & Environmental Sources \\
\hline $\begin{array}{l}\text { heptane, } \\
\text { 1-propanol, } \\
\text { methanol, } \\
\text { toluene, } \\
\text { vegetable oil }^{1}\end{array}$ & $\begin{array}{l}\text { paper, cotton, } \\
\text { polystyrene, } \\
\text { pine, cardboard, } \\
\text { cheesecloth, } \\
\text { toast }^{2}\end{array}$ & propane & $\begin{array}{l}\text { propane, aerosols (disinfectant, } \\
\text { furniture polish, cooking spray, hair } \\
\text { spray), nail polish remover, ammonia- } \\
\text { based window cleaner, bleach, water } \\
\text { mist, boiling water, toast, cigarette } \\
\text { smoke, coffee }\end{array}$ \\
\hline
\end{tabular}

The method of generating the airborne signatures varied for each of the categories of sources. A detailed description of the methods and is provided by Hagen [7]. Nuisance sources were generated by methods considered to be typical, e.g. toast was overcooked in a household toaster and aerosol sprays were continuously sprayed while randomly walking around the room.

Measurements describing the mass loss and signatures of the sources are documented by Hagen [7]. The signature data is reviewed for the purpose of identifying patterns associated with the categories of sources. General trends are noted from a manual review of the maximum values recorded for each sensor leading to the development of another elementary 
expert system similar to that developed for the data from the small-scale tests. This expert system provides insight into the patterns present in the experiments.

A multivariate statistical analysis is applied to the maximum values recorded for each sensor during each test to identify the nature of the source instead of a neural network. Given the limited number of sensors, the same level of classification can be obtained with a less complex method than a neural network. The type of statistical analysis, a principal component analysis (PCA), makes use of the maximum values measured by each sensor for each test. The data are arranged in a data matrix, $\mathbf{X}[8,10-12]$. PCA determines the linear combinations of the maxima that are capable of explaining most of the variations in the measurements. Specifically, the PCA approach seeks to maximize:

$$
\mathrm{p}_{1}^{\mathrm{T}} \mathrm{X}^{\mathrm{T}} \mathrm{Xp}_{1}
$$

with the constraint of $\mathrm{p}_{1}^{\mathrm{T}} \mathrm{p}_{1}=1$.

$\mathrm{p}_{1}$ is a matrix of coefficients, referred to as the principal components. As expressed, the solution of equation (1) yields the most important direction, $\mathrm{p}_{1}$, of the maximum variation of the data. Equation (1) is then re-expressed to determine the second most important direction, $\mathrm{p}_{2}$, etc. The number of coefficients selected for the PCA approach is based upon using the least number of principal components which provide sufficiently accurate predictions. For this application, an accuracy of 75-80 percent was judged acceptable.

The predictions of the data are provide by a product of the principal components matrix, $\mathrm{P}$, with a score matrix, $\mathrm{T}$, as presented in equation (2).

$$
\mathrm{X}=\mathrm{TP}^{\mathrm{T}}
$$

The column vectors in the $\mathrm{P}$ and $\mathrm{T}$ matrices are required to be orthogonal, i.e.:

$$
\begin{aligned}
& \mathrm{t}_{\mathrm{i}} \mathrm{t}_{\mathrm{j}}=\left\{\begin{array}{l}
1, \mathrm{i}=\mathrm{j} \\
0, \mathrm{i} \neq \mathrm{j}
\end{array}\right. \\
& \mathrm{p}_{\mathrm{i}} \mathrm{p}_{\mathrm{j}}=\left\{\begin{array}{l}
1, \mathrm{i}=\mathrm{j} \\
0, \mathrm{i} \neq \mathrm{j}
\end{array}\right.
\end{aligned}
$$

The score matrix, $\mathrm{T}$, is determined by:

$$
\mathrm{T}=\mathrm{XP}
$$

Consequently, a linear combination of scores, $t_{i}$, and coefficients, $p_{i}$, are used to reconstruct the raw sensor measurements, as expressed in equation (4) for three principal components.

$$
X=t_{1} p_{1}+t_{2} p_{2}+t_{3} p_{3}
$$


Measurements from the following six sensors used in the tests are applied to develop the PCA model: $\mathrm{CO}, \mathrm{CO}_{2}$, two Taguchi sensors (T880 and T822), temperature and light obscuration. The data for each sensor is scaled to zero mean and unit variance. The data collected from each sensor prior to the introduction of the source is used to establish normal, background conditions for that test. Characterizing ambient conditions was especially important given the unconditioned nature of the test room. Three PCA components explain approximately $76 \%$ of the variability in the ambient data (collected two minutes prior to the introduction of any source). Consequently, three components are used to classify the sources.

The squared difference between the raw sensor values and the reconstructed values is called the squared prediction error (SPE). The expression for the SPE with three principal components is:

$\operatorname{SPE}=\left(x_{i} \pm t_{1} p_{1} \pm t_{2} p_{2} \pm t_{3} p_{3}\right)^{2}$

Both the scores, $t_{i}$, and the SPE reflect all of the sensor measurements because both the scores and the SPE involve data compression as well as synthesis. The SPE is used to identify the existence of an abnormal situation, with its confidence limit set at $99.5 \%$. Where a set of measurements exceed the SPE outside of the established limit in three successive scans (with a scan rate of $2 \mathrm{sec}$. for all measurements), the identified conditions are considered to be "abnormal". The SPE successfully identifies conditions generated in all 87 tests as differing from normal conditions.

Following identification of abnormal conditions, the PCA is applied to classify the nature of the source. The scores $\left(t_{i}\right)$ are used to distinguish the type of source, using the following rules:

- if $t_{3}>5$, then the source is a flaming fire

- if $-8<t_{2}<0$, then the source is a smoldering fire

- otherwise the source is a nuisance source.

The results of applying the above rules are summarized in Table 2 for the test data. All of the flaming sources are properly classified, with smoldering sources classified properly in $88 \%$ of the tests. Nuisance and ambient sources are classified properly in $73 \%$ of the tests by the PCA evaluation of the sensor data. $27 \%$ of the nuisance source cases are incorrectly identified as smoldering sources and hence represent false alarms. In contrast, at least one of the commercial detectors respond to $97 \%$ of the flaming fires (one is missed) and $25 \%$ of the non-flaming fires. The commercial detectors also respond to $11 \%$ of the nuisance sources as false alarms.

In addition to the improved classification rate, the time for detection of the signatures from fire sources is significantly less with the measurements included and the PCA-based intelligence than that for either of the commercial detectors. The time required for detection of flaming fires is reduced by an average of $45 \mathrm{~s}$ (representing a decrease of 57\%), with the detection time for the PCA-based evaluation of the data being 6 to $244 \mathrm{~s}$ less than that for the 
first responding commercial detector. The decrease in detection time was greater for the nonflaming fires, having an average reduction of $245 \mathrm{~s}$ and a range of 182 to $332 \mathrm{~s}$.

\section{TABLE 2 Classification of Test Sources}

\begin{tabular}{|c|c|c|c|c|c|}
\hline & \multicolumn{3}{|c|}{ Classification } & \multicolumn{2}{|c|}{ Summary } \\
\hline & Flaming & Smoldering & $\begin{array}{l}\text { Nuisance/ } \\
\text { Ambient }\end{array}$ & Total & $\%$ Correct \\
\hline Flaming Fire & 34 & & & 34 & 100 \\
\hline $\begin{array}{l}\text { Smoldering } \\
\text { Fire }\end{array}$ & & 14 & 2 & 16 & 88 \\
\hline $\begin{array}{l}\text { Nuisance/ } \\
\text { Ambient }\end{array}$ & & 10 & 27 & 37 & 73 \\
\hline Total & & & & 87 & 86 \\
\hline
\end{tabular}

\section{Mixed Source Experiments}

The variety of sources used in the third phase to generate conditions from combinations of sources within the room are summarized in Table 3 [9]. The set of sources selected represent a sample of the range of sources used in the second phase. A set of baseline tests are conducted using each of the sources alone. The methods of introduction for the fire and non-fire sources is similar to that used in the second phase, with a detailed description of each method provided by Hopkins [9]. In general, the combinations are produced by recording ambient conditions for two minutes, followed by the introduction of the nuisance source for $90 \mathrm{~s}$ throughout the room. Except for the case with boiling water which is continued along with the fire source, the nuisance source is discontinued and the fire source initiated.

\section{TABLE 3 Combinations of Fire and Non-Fire Sources}

\begin{tabular}{|l|c|c|c|c|c|}
\hline & \multicolumn{5}{|c|}{ Non-Fire Sources } \\
\hline Fire Sources & None & $\begin{array}{c}\text { Aerosol } \\
\text { Disinfectant }\end{array}$ & $\begin{array}{c}\text { Window } \\
\text { Cleaner }\end{array}$ & $\begin{array}{c}\text { Aerosol } \\
\text { Hairspray }\end{array}$ & $\begin{array}{c}\text { Boiling } \\
\text { Water }\end{array}$ \\
\hline None & - & $\mathrm{x}$ & $\mathrm{x}$ & $\mathrm{x}$ & $\mathrm{x}$ \\
\hline Heptane & $\mathrm{x}$ & $\mathrm{x}$ & $\mathrm{x}$ & $\mathrm{x}$ & $\mathrm{x}$ \\
\hline Paper & $\mathrm{x}$ & $\mathrm{x}$ & $\mathrm{x}$ & $\mathrm{x}$ & $\mathrm{x}$ \\
\hline Cloth & $\mathrm{x}$ & $\mathrm{x}$ & $\mathrm{x}$ & $\mathrm{x}$ & $\mathrm{x}$ \\
\hline Hamburger & $\mathrm{x}$ & $\mathrm{x}$ & $\mathrm{x}$ & $\mathrm{x}$ & $\mathrm{x}$ \\
\hline
\end{tabular}


As with the previous phase involving single sources, the mass loss rate for the fire sources is relatively modest, e.g. an average mass loss rate for the heptane fires was $0.3 \mathrm{~g} / \mathrm{s}$. The aerosol and window cleaner are introduced by continuously engaging the button on the aerosol can or the dispenser of the window cleaner. Water is evaporated at an approximate rate of $15 \mathrm{ml} / \mathrm{s}$ and a hamburger (nominal mass of $100 \mathrm{~g}$ ) is cooked on a hotplate.

The history of the ratio of $\mathrm{CO}$ and $\mathrm{CO}_{2}$ concentrations for each of the tests with heptane is presented as Figure 2. The curves for the combination sources have been shifted such that zero time is associated with ignition of the heptane, following the $90 \mathrm{~s}$ introduction of the nuisance source. As indicated in the figure, the difference in the ratio for the case with the flaming heptane alone and the cases with the flaming heptane and the additional sources is relatively modest. The greatest value of the ratio is obtained for the aerosol spray, which contained an assortment of hydrocarbons. The average $\mathrm{CO} / \mathrm{CO}_{2}$ ratio for the entire duration of the test for the other fire sources with the nuisance sources is presented in Table 4.

\section{FIGURE $2 \mathrm{CO} / \mathrm{CO}_{2}$ Ratio for Heptane and Combination Sources Including Heptane}

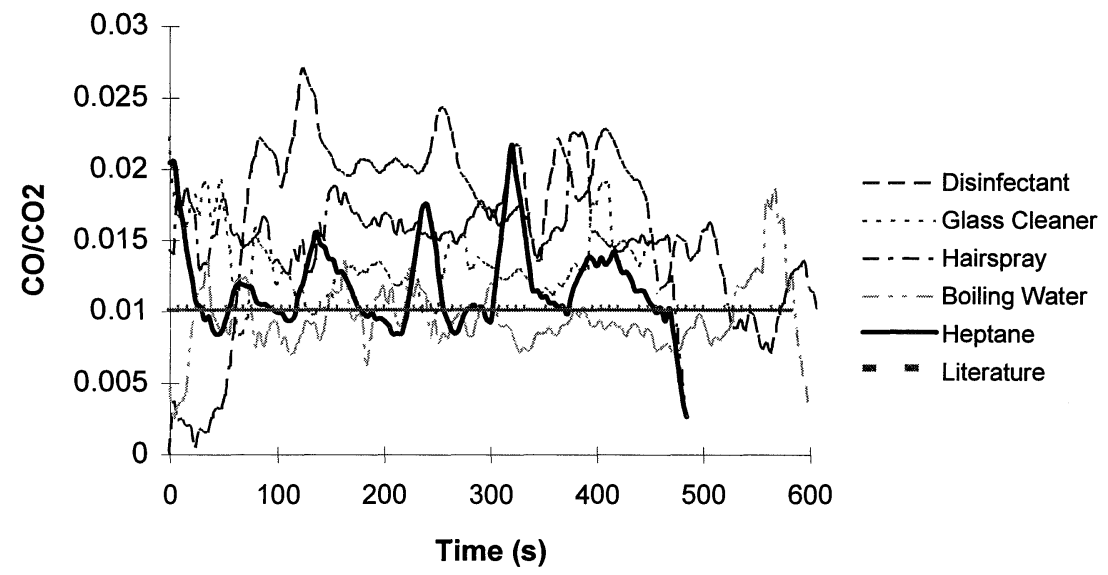

As indicated in the table, an elementary expert system can be proposed based only on the $\mathrm{CO} / \mathrm{CO}_{2}$ ratio to distinguish between flaming fire and non-flaming fire sources, given the limited data available. Support for this system is based on the observation that each of the fire sources appears to have a characteristic $\mathrm{CO} / \mathrm{CO}_{2}$ ratio, as is confirmed in the literature for a wider range of fuels and burning modes [13]. The range of the $\mathrm{CO} / \mathrm{CO}_{2}$ ratio for all of the combinations involving heptane and the flaming paper fires is 0.01 to 0.14 , while the ratio for the pyrolyzing cotton is significantly greater at 0.23 to 0.49 . 
TABLE 4 Average $\mathrm{CO} / \mathrm{CO}_{2}$ Ratio for Fire and Nuisance Source Combinations

\begin{tabular}{|l|c|c|c|c|c|}
\hline & \multicolumn{5}{|c|}{ Nuisance Source } \\
\hline Fire Source & None & Disinfectant & $\begin{array}{c}\text { Window } \\
\text { Cleaner }\end{array}$ & Hairspray & $\begin{array}{c}\text { Boiling } \\
\text { Water }\end{array}$ \\
\hline Heptane & 0.01 & 0.02 & 0.01 & 0.02 & 0.01 \\
\hline Flaming Paper & 0.11 & 0.09 & 0.08 & 0.14 & 0.06 \\
\hline Pyrolyzing Cotton & 0.37 & 0.24 & 0.49 & 0.29 & 0.23 \\
\hline
\end{tabular}

However, the $\mathrm{CO} / \mathrm{CO}_{2}$ ratio for the variety of combinations involving hamburger ranged from 0.20 to 0.30 . Consequently, an expert system based only on the $\mathrm{CO} / \mathrm{CO}_{2}$ ratio will yield unnecessary alarms for the combination sources with hamburger. As a result, discrimination of the non-flaming and nuisance sources requires the use of additional sensors. The average and maximum values of the signals received from the metal oxide sensors are not easily categorized for the variety of multiple sources. After the period of introduction of the nuisance source, the two metal oxide sensors responded differently to the combined signature. The response of one sensor approached that of the heptane alone, while the other appeared to reach an average value of response for heptane alone and the nuisance source alone. These differences are attributable to the inherent characteristics of each sensor. However, the difficulty with these different sensors is that a simplistic method of discrimination suggested in the second phase using only threshold values is not appropriate. Consequently, a method of discrimination is being investigated which considers transient data (rather than just maximum values) to overcome the tendency of the maximum value algorithm to be easily tricked by the non-fire sources.

\section{SUMMARY}

As a result of the experimental effort, an early fire detector consisting of an array of six sensors appears feasible, with discrimination provided by a neural network or multivariate statistical analysis of the sensor responses. The PCA-based evaluation of the sensor data described has the ability to respond more quickly as compared to currently available commercial detectors. Additional research is necessary to characterize the signatures from scenarios involving additional combination sources which can mask fire signatures or cause unnecessary alarms. In addition, the merits of a more comprehensive characterization of the signatures of fire and non-fire sources through the use of additional sensors or the use of transient data should be investigated. This analysis should improve the discrimination ability between flaming fire, non-flaming fire and nuisance sources.

\section{ACKNOWLEDGMENTS}

This project is supported by the Building and Fire Research Laboratory of the National Institute of Standards and Technology (NIST). Dr. William Grosshandler is the technical monitor. Appreciation is extended to the graduate students who have contributed to this 
project, including S. Denny, B. Hagen, and M. Hopkins in Fire Protection Engineering and D. Pan and T. Kunt in Chemical Engineering.

\section{SELECTED REFERENCES}

[1] Hall, J.R., "The Latest Statistics on U.S. Home Smoke Detectors," Fire J., 83: 1, 39-41, 1989.

[2] Thuillard, M., "New Methods for Reducing the Number of False Alarms in Fire Detection Systems," Fire Technology, 30: 2, 250-268, 1994.

[3] Denny, Samuel, "Development of a Discriminating Fire Detector for Use in Residential Occupancies," Report FP 93-07, M.S. Thesis, College Park, Fire Protection Engineering, University of Maryland, December 1993.

[4] Okayama, Y., "Approach to Detection of Fires in Their Very Early Stage by Odor Sensors and Neural Net", Proceedings of the 3rd International Symposium of Fire Safety Science, p. 955-964, 1991.

[5] Pan, D., "Applications of Pattern Recognition Using Neural Networks," M.S. Thesis, College Park, Chemical Engineering, University of Maryland, 1994.

[6] Milke, J.A. and McAvoy, T.J., "Analysis of Signature Patterns for Discriminating Fire Detection with Multiple Sensors," Fire Technology, 31:3, 120-136, 1995.

[7] Hagen, B.C., "Evaluation of Gaseous Signatures in Large-Scale Test," Report FP 94-05, M.S. Thesis, College Park, Fire Protection Engineering, University of Maryland, December 1994.

[8] McAvoy, T.J., Milke, J., and Kunt, T.A., "Using Multivariate Statistical Methods to Detect Fires," Fire Technology, 32:1, 6-24, 1996.

[9] Hopkins, Mark, "A Study of Gaseous Signatures in Large-Scale Tests with Multiple Source Scenarios,” M.S. Thesis, College Park, Fire Protection Engineering, University of Maryland, May 1996.

[10] Dong, D. and McAvoy, T.J., "Nonlinear Principal Component Analysis - Based on Principal Curves and Neural Networks," Computers and Chemical Engineering, 20: 1996, 65-78.

[11] Kresta, J., MacGregor, J., and Marlin, T., "Multivariate Statistical Monitoring of Process Operating Performance," Canadian J. Chemical Engineering, 69: 1991, 35-47.

[12] Nomikos, P., and MacGregor, J., "Monitoring Batch Processes Using Multi-way PCA," AIChE J, 40: 1994, 1361-1375.

[13] Tewarson, A., "Generation of Heat and Chemical Compounds in Fires," in SFPE Handbook of Fire Protection Engineering, 2nd edition, NFPA, p. 3-53 to 3-124, 1995. 